\title{
SISTEMA DE BAJO COSTO PARA LA PROGRAMACIÓN POR DEMOSTRACIÓN DEL AGARRE DE OBJETOS EN ROBÓTICA
}

\section{LOW-COST SYSTEM FOR PROGRAMMING BY DEMONSTRATION GRASP OF OBJECTS IN ROBOTICS}

José Hoyos-Gutiérrez ${ }^{1}$, Carlos Peña ${ }^{2}$, Flavio Prieto², Pedro Cárdenas², Manuel Núñez².

${ }^{1 .}$ Facultad de Ciencias Básicas y Tec., Universidad del Quindío, Colombia josegabrielh@uniquindio.edu.co

2. Facultad de Ingeniería, Universidad Nacional de Colombia, Colombia.

Recibido: 07 de febrero de 2014

Aceptado: 18 de marzo de 2014

*Correspondencia del autor: Email: josegabrielh@uniquindio.edu.co

\section{RESUMEN}

En este trabajo se presenta la implementación y resultados de pruebas preliminares de un sistema de bajo costo, que permite la captura de las señales implicadas en el agarre de objetos en robótica. El sistema está compuesto de un guante de datos y una mano robótica de tres dedos. El guante de datos es de fabricación propia y contiene los sensores de posición y fuerza. Las señales obtenidas por estos sensores, son almacenadas para su posterior reproducción. La mano está desarrollada en prototipado rápido. El sistema desarrollado es de bajo costo comparado con los comerciales. Además puede ser fácilmente reproducible, ya que se usan elementos comerciales. A través de algunos resultados, se muestra el funcionamiento del sistema desarrollado.

Palabras Clave: Guante de datos, sensor de fuerza, sensor de flexión

\begin{abstract}
We present the implementation and results obtained from preliminary tests in a low-cost system, which allows the signals acquisition of the grasp of objects in robotics. The system is composed of a data glove and a robotic hand with three fingers. The data glove is our own make and it has force and position sensors. The signals obtained are saved for later reproduction. The robotic hand was developed with rapid prototyping. The developed system presents low cost compared with the commercial systems. Besides, it can be easily duplicated because of the commercial elements that compose it. The results show the operation of the developed system.
\end{abstract}

Keywords: Data glove, force sensor, flexion sensor. 


\section{INTRODUCCIÓN}

En este trabajo se presenta la implementación y resultados de pruebas preliminares a un sistema de bajo costo, el cual permite la captura de las señales implicadas en el agarre de objetos en robótica.

El problema de agarrar objetos en robótica, se puede dividir en tres partes: i) Orientar y posicionar la mano en una forma adecuada, ii) Cerrar la mano para que el objeto, al ser tomado, permanezca firmemente agarrado, y iii) Dosificar la fuerza para que el objeto no sea destruido, si se trata de un objeto frágil.

Existen dos tipos de agarre: el agarre de potencia, donde se utiliza la palma de la mano y el cierre de los dedos para sujetar el objeto, y el agarre de precisión, donde el agarre se realiza utilizando las yemas de los dedos (1).

El aprendizaje por imitación (ApI) es un conjunto de técnicas, donde al contrario de la programación detallada que se hace al robot convencionalmente, este aprende movimientos parciales o incluso una tarea completa, con los datos obtenidos de una o varias demostraciones de los movimientos o la tarea por parte de un humano.

El aprendizaje por imitación permite la flexibilidad en la programación de tareas que puede realizar un robot humanoide o un robot industrial, ya que puede ser instruido por operarios sin conocimientos en programación de robots.

Los primeros trabajos en aprendizaje por imitación son llamados programación por demostración y datan de los años 80s, como lo expresan Schaal (2), Calinon (3) y Argall (4). Estas técnicas surgen como una posible solución, o ruta alterna, que evita la tarea de programar el robot para tareas complejas. El aprendizaje por imitación fue estudiado inicialmente en psicología, donde se relacionan los comportamientos de aprendizaje con áreas del cerebro, por ejemplo en Schaal (2) y Oztop (5), se presenta un modelo del cerebro de un simio, donde se relacionan partes del cerebro con las capacidades de visión y motrices. También, desde el punto de vista cognitivo, Breazeal y Scassellati (6) presentan una clasificación del aprendizaje social, donde lo dividen en: imitación, emulación de metas, mejora por estímulo, apoyo social, exposición en ambiente, facilitación social, etc.

El sistema presentado en este documento está compuesto de un guante de datos y una mano robótica con tres dedos. El guante de datos es de fabricación propia y contiene los sensores de posición y fuerza. Las señales obtenidas por estos sensores son almacenadas para su posterior reproducción. La mano está basada en la desarrollada en el instituto KTH de Suecia (7) y fue realizada con prototipado rápido en piezas de plástico. Consta de servomotores, sensores de posición magnético y sensores de fuerza, y es controlada desde un PC a través de un microcontrolador de 32 bits.

El sistema desarrollado es de bajo costo comparado con los comerciales. Además puede ser fácilmente duplicado, ya que se usan elementos comerciales. A través de algunos resultados, se muestra el funcionamiento del sistema.

\section{DESARROLLO DEL TEMA}

En esta sección se presenta la implementación de los elementos constituyentes del sistema. Primero se presenta la implementación de la mano robótica. A continuación, el desarrollo del guante para la adquisición de datos.

\section{Instrumentación}

En la Figura 1 se presenta la parte interna de la mano robótica. En la Figura 2 se presenta un esquema del sistema implementado.

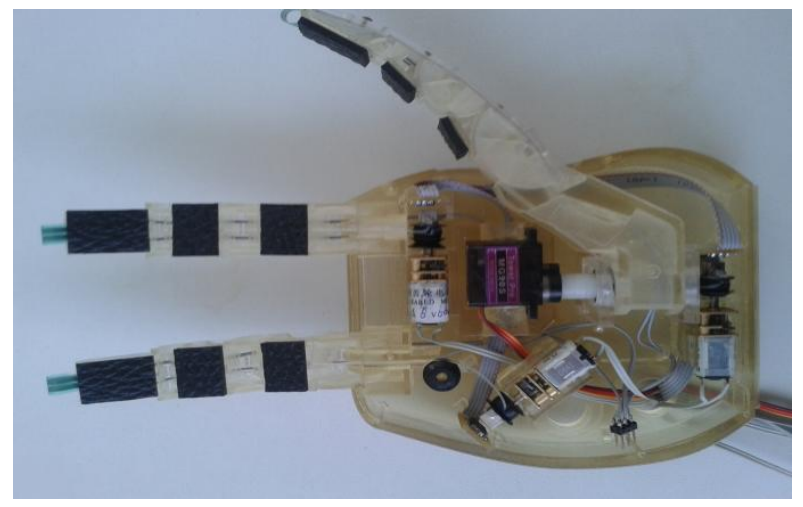

Figura 1. Detalle de la mano robótica

La mano está compuesta de varios sensores, que permiten medir la fuerza en la yema de los dedos (sensor FSR) y el ángulo de curvatura en los dedos, el cual se mide indirectamente en el eje de los motores con un encoder absoluto. El sensor FSR es un polímero conductor que cambia su resistencia de manera predecible al aplicar una fuerza en su superficie. Los sensores de posición son implementados a través de un circuito integrado AS5040, el cual mide variaciones de campo magnético.

Entre los circuitos electrónicos se encuentran varios puente $\mathrm{H}$, la adecuación de la señal de los sensores 
FSR, y la tarjeta de control y comunicación con el computador, la cual se implementó usando la tarjeta de desarrollo STM 32F4 DISCOVERY. En esta tarjeta se programaron las funciones para la entrada de información de los sensores encoder absoluto (SPI), sensor FSR (ADC), corriente en los motores (ADC) y salida para los motores (PWM).

La función del SPI es recibir los datos provenientes de los encoders absolutos, obteniendo de forma serial las posiciones de los ejes de los tres moto-reductores. En este caso no es necesario adecuar la señal, ya que el microcontrolador la lee directamente por un puerto dado que los tres encoders se encuentran conectados en cascada. La función del ADC es registrar los cambios en los sensores de fuerza mediante un divisor de tensión; además, se encarga de registrar la corriente que es suministrada a cada uno de los motores a través de una resistencia de sondeo sobre cada puente-H.

La salida PWM permite manejar las señales para el control de velocidad de los moto-reductores y el servomotor.

\section{Guante de datos}

Está compuesto por sensores de fuerza y flexión. Los primeros miden la fuerza en la yema de los dedos mientras los sensores de flexión miden el ángulo de las articulaciones de los dedos.

Se realizó una preselección de los sensores a comparar, basada en la disponibilidad. La selección final se realizó según criterios como el área de sensado, la sensibilidad, y el precio, como se observa en la Tabla 1.

Se eligió el sensor FSR (Force Sensitive Resistor) de 0.5 ", dado que el rango de fuerza permite la lectura de datos entre $100 \mathrm{~g}$ y $10 \mathrm{~kg}$, y se propuso la manipulación de objetos con pesos en el rango estipulado.

La selección se realizó teniendo en consideración también el precio, el cual es tres veces menor al precio de los demás sensores.

Es importante anotar que la selección por precio se realiza comprometiendo la precisión del sensor. Debido a que se planea utilizar programación por demostración, se considera que una alta precisión en los datos no es necesaria.

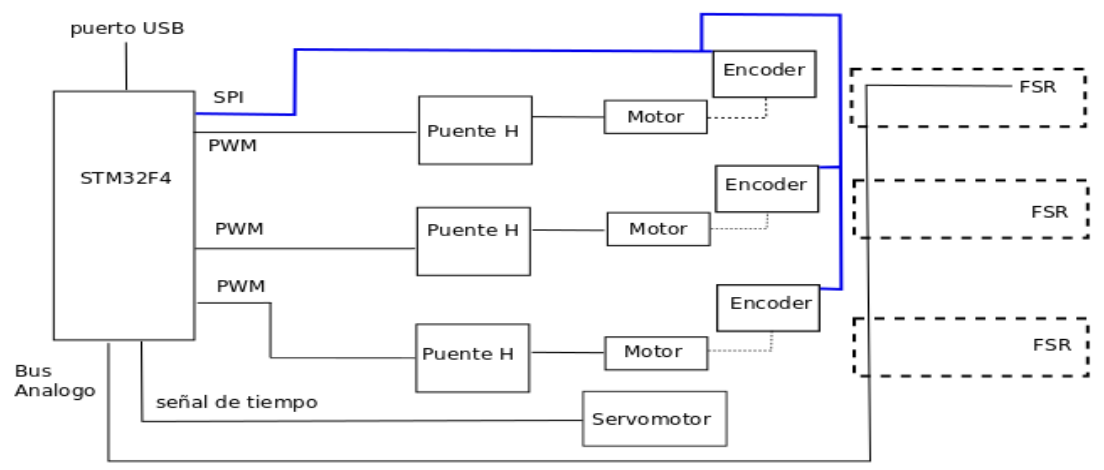

Figura 2. Esquema de bloques de la mano

Tabla 1. Características utilizadas en la selección del sensor de fuerza.

\begin{tabular}{|l|c|c|c|}
\hline Característica & $\begin{array}{c}\text { Standard Force } \\
\text { and } \\
\text { Load Sensors } \\
\text { Model \#A201 }\end{array}$ & $\begin{array}{c}\text { Standard Force } \\
\text { and } \\
\text { Load Sensors } \\
\text { Model \#A301 }\end{array}$ & $\begin{array}{c}\text { Force Sensitive } \\
\text { Resistor } \\
\mathbf{0 . 5} \text { "SEN-09375 }\end{array}$ \\
\hline Longitud total [mm] & 197 & 25.4 & 60.3 \\
\hline Ancho total [mm] & 14 & 14 & 19 \\
\hline Diámetro de detección $[\mathrm{mm}]$ & 9.53 & 9.53 & 12.7 \\
\hline Rango de fuerza [lb] & $0-100$ & $0-100$ & $0-25$ \\
\hline Precio [pesos colombianos] & 60900 & 63800 & 16000 \\
\hline
\end{tabular}


Se determinó la actividad de agarre a realizar como un agarre de precisión, el cual se realiza utilizando las puntas de los dedos. Además, se definió el uso de tres sensores: el primero en la yema del dedo pulgar, el segundo en la yema del dedo índice y el tercero en la yema del dedo medio (Figura 3).

La elección se realizó teniendo en cuenta que son los dedos más utilizados al momento de agarrar objetos de forma precisa.

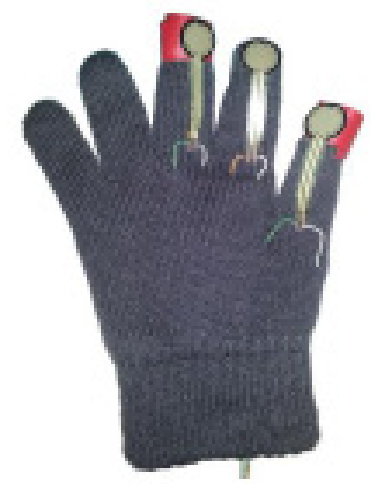

Figura 3. Guante con los sensores de fuerza instalados (vista palmar).

Además de la información de fuerza, se obtuvo la flexión o curvatura de los dedos utilizando sensores de flexión FS7954. La instalación se realizó colocando bolsillos sobre el guante que aseguran que el sensor esté ubicado sobre las articulaciones, como se observa en la Figura 4.

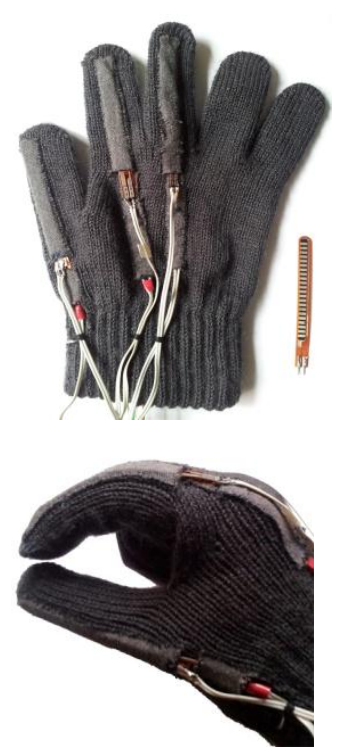

Figura 4. Guante con los sensores de flexión instalados Arriba: vista dorsal. Abajo: vista lateral.
Para cada uno de los dedos pulgar, índice y medio se mide la flexión de dos articulaciones, la articulación metacarpofalangiana (MCF) y la articulación interfalangiana proximal (IFP) (Figura 5).

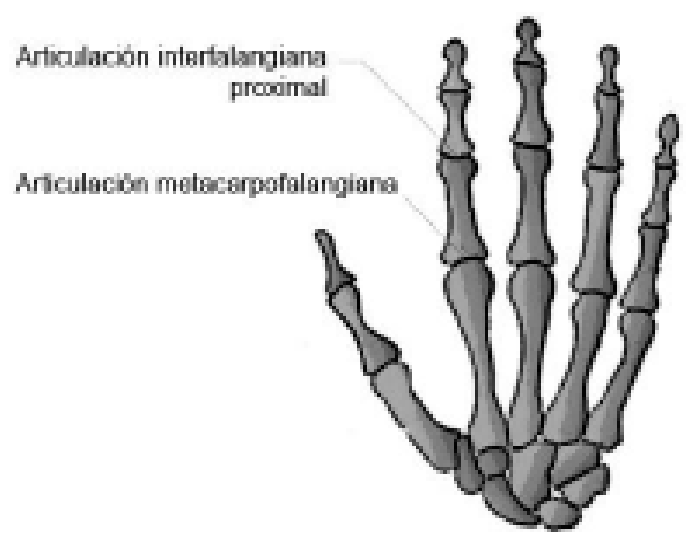

Figura 5. Articulaciones medidas en los dedos pulgar, índice y medio.

Cada tipo de sensor requiere circuitos de acondicionamiento. Para el sensor de flexión se implementó un conversor resistencia a voltaje (Figura 6a), mientras que el sensor de fuerza se acopló mediante un divisor de voltaje que lo protege de sobrecorrientes (Figura 6b). Para la adquisición de los datos se utilizó una tarjeta National Instruments NI-USB 6008.

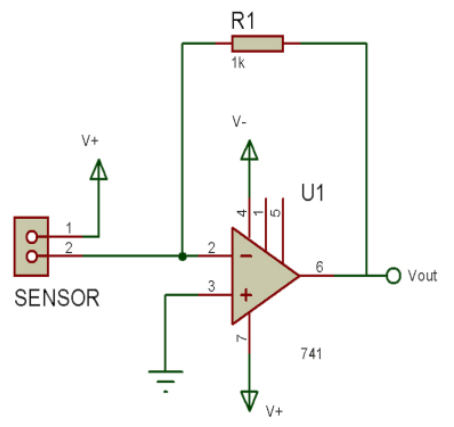

a)

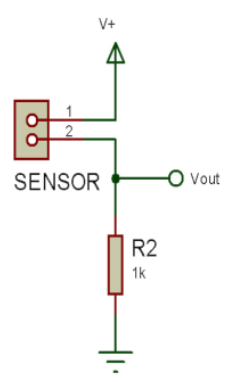

b)
Figura 6. Circuitos de acondicionamiento. a) Sensor de flexión b) Sensor de fuerza.

\section{RESULTADOS Y DISCUSIÓN}

Se presentan los resultados obtenidos al probar el funcionamiento del guante de datos al agarrar tres objetos de diferentes dimensiones y pesos. Además, una secuencia de la mano robótica tomando un envase plástico.

\section{Pruebas preliminares con el guante}

Se obtuvieron los datos de voltaje de los sensores de fuerza al sostener con un agarre fino tres objetos diferentes, con lo cual se realizaron las gráficas presentadas en las Figuras $7-9$. 
En las tres figuras se presentan los voltajes de los dedos pulgar, índice y medio en triángulos rojos, equis verdes y puntos azules, respectivamente.

En la Figura 7, se realiza el agarre de un celular desde sus bordes laterales, con el dedo pulgar debajo del objeto y los dedos índice y medio sobre este.

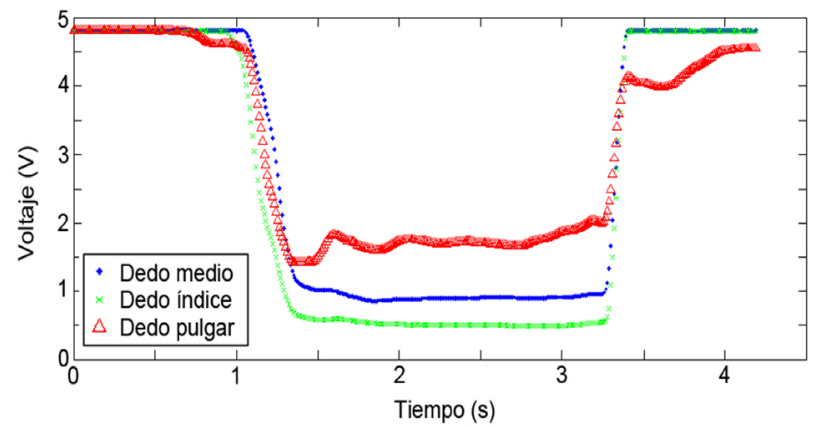

Figura 7. Voltaje obtenido de los sensores de fuerza del agarre de un celular.

En la Figura 8, se realiza el agarre de un estuche de CD cuadrado y vacío, con el dedo pulgar debajo del objeto y los dedos índice y medio sobre este.

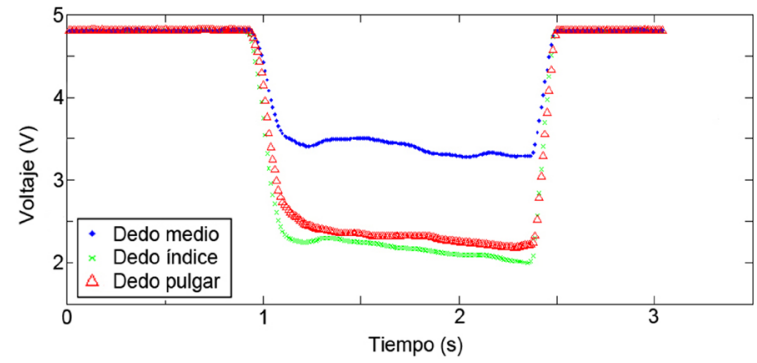

Figura 8. Voltaje obtenido de los sensores de fuerza del agarre de un estuche de CD.

En la Figura 9, se realiza el agarre de un mando del nintendo Wii desde sus bordes laterales, con el dedo pulgar debajo del objeto y los dedos índice y medio sobre este.

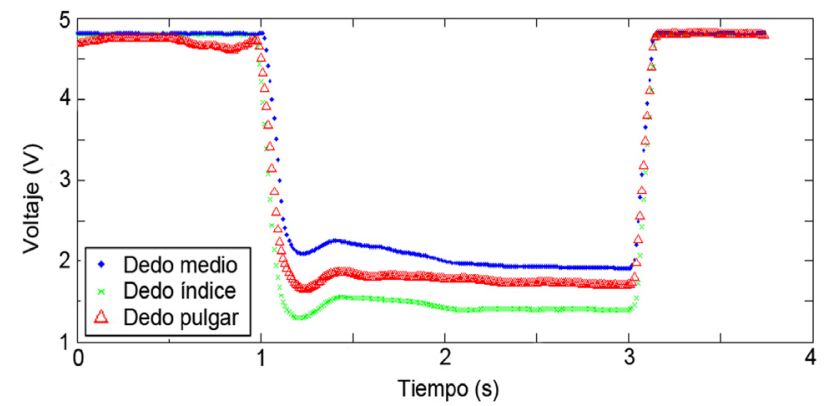

Figura 9. Voltaje obtenido de los sensores de fuerza del agarre de un mando de Wii.

Aunque en las tres pruebas la mayor variación se presenta en el dedo índice, el valor depende de la ubicación de los dedos sobre el objeto. Si ambos dedos se encuentran equidistantes al centro de gravedad de este, el valor medido sería igual en ambos.
Por su parte, el dedo pulgar, al encontrarse debajo de los objetos, presenta una variación proporcional al peso del mismo.

Para probar los sensores de flexión se realizó un agarre de precisión de una esfera pequeña, obteniendo la Figura 10, donde se presenta cada dedo en un eje separado. La línea sólida azul es el voltaje obtenido por el sensor sobre la articulación MCF, mientras que la línea punteada roja es el valor en la articulación IFP. En la Figura 11, se muestra la secuencia de la mano robótica tomando una botella plástica. La reproducción se realiza a partir de datos obtenidos con el guante de datos al agarrar el mismo objeto.
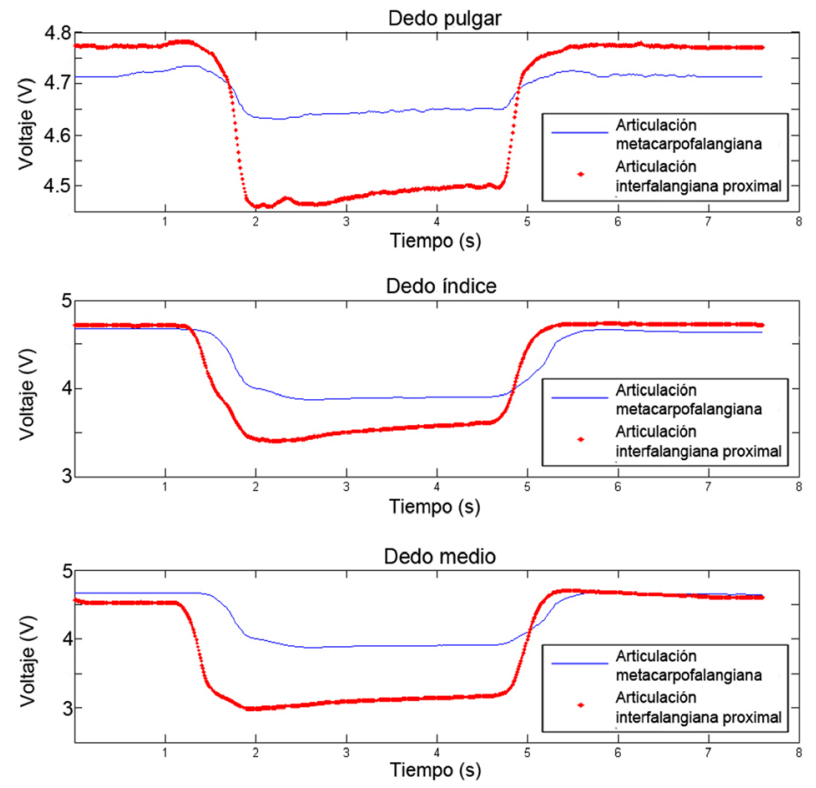

Figura 10. Voltaje obtenido de los sensores de flexión del agarre de una esfera pequeña.

\section{CONCLUSIONES}

En este artículo se presentó, la implementación y pruebas de un sistema de bajo costo, para el estudio de agarre de objetos en programación por demostración. El desarrollo de sistemas utilizando circuitos integrados y sensores de bajo costo como los utilizados en este proyecto, permite la implementación de sistemas robóticos de menor valor, aunque su precisión no sea tan alta como en los sistemas comerciales.

Al utilizar sensores de fuerza y flexión, es importante tener en cuenta el tipo de agarre que se está realizando, así como la ubicación de los dedos sobre el objeto. De esta forma, se asegura que la reproducción de los datos sobre la mano robótica sea confiable y la información se utilice de manera correcta.

Como trabajo futuro, se planea integrar la mano ro- 
bótica en un manipulador de 6 grados de libertad, además de agregar la etapa de aprendizaje por imitación para la codificación de los datos obtenidos con el guante de datos.
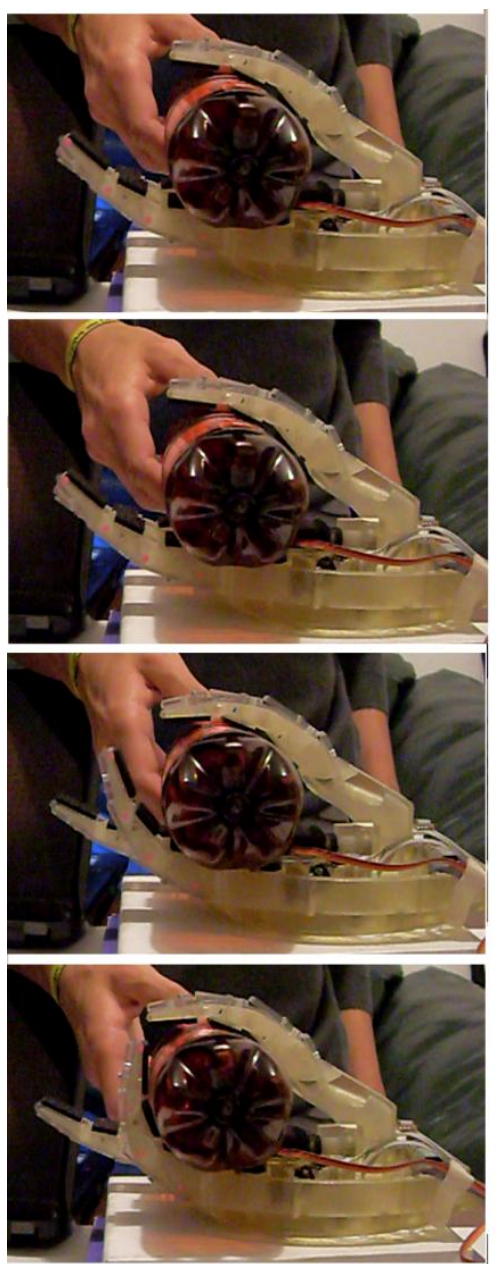

Figura 11. Secuencia del agarre de una botella plástica.

\section{BIBLIOGRAFÍA}

1. Cutkosky, M. R. "On grasp choice, grasp models, and the design of hands for manufacturing tasks". En IEEE Transactions on Robotics and Automation, 1989; 5(3), 269-279.

2. Schaal, S. "Is imitation learning the route to humanoid robots?" En Trends in cognitive sciences, 1999; 3(6), 233-242.

3. Calinon, S. "Robot programming by demonstration". En Springer Handbook of Robotics 2008; (13711394). Springer Berlin Heidelberg.

4. Argall, B. D., Chernova, S., Veloso, M., \& Browning, B. "A survey of robot learning from demonstration". En Robotics and Autonomous Systems, 2009; 57(5), 469-483.

5. Oztop, E., Kawato, M., \& Arbib, M. "Mirror neurons and imitation: A computationally guided review". En Neural Networks, 2006; 19(3), 254-271.

6. Breazeal, C., \& Scassellati, B. "Robots that imitate humans". En Trends in Cognitive Sciences, 2002; 6(11), 481-487.

7. Schmidt Klaus. "Robot Hand Design -PALM Improvement and A Robotic Hand-Arm Interface". Thesis KTH, Royal institute of Thecnology. 2008. 